\title{
Relaunched, the Australian Journal of Advanced Nursing in the year of the Nurse and Midwife
}

Two thousand and twenty; the year that was. The World Health Organization-designated Year of the Nurse and Midwife was in no way celebrated as intended but could not have highlighted more pertinently the global importance of the nursing and midwifery professions. Although challenging for all, we may also reflect that it was a year of many successes for the Australian Journal of Advanced Nursing. Successes which can be credited to the authors, peer reviewers, and readers who throughout the year committed their time and expertise to ensuring an excellent volume of issues. We are consistently humbled by the quality and clear effort of the contributions made to the journal.

Beginning the year with the relaunch of the journal's website, the platform through which four quarterly issues were published, we have seen contributions to research and discussion covering a vast array of relevant topics, from the role of rural and remote nurses in Australia, to the development of nurse-led models of care facilitating coordinated outpatient care for those with multimorbidity. As well as research reports, we have also received and published several guest editorials from some leading experts in nursing, health, and aged care that provide important points of view on critical issues including residential aged care in Australia and of course COVID-19. Beyond publishing, we have also expanded the Editorial Board, with a panel of nurses, midwives and academics providing a diversity of national and international knowledge and experience. Finally, in our ongoing effort to support authors, we released revised author guidelines which we hope provide clear direction throughout the process of manuscript development and submission.

The work however is not done. In 2021 we hope to continue improving our guidance for authors and peer reviewers and release further supportive materials. We will continue to expand the Editorial Team, welcoming new Associate Editors to handle author manuscripts through peer review. From authors, we also hope to receive submissions to some of the newer sections of the journal. This includes a section looking to capture interesting and relevant case studies - shorter manuscripts reporting work which may not present suitably as empirical research, but which provide a unique commentary on issues related to the Journal's mission and scope. Further, we invite Letters to the Editor, be they in response to published articles, or as independent works providing compelling commentary or debate to the readership. Finally, we plan to put out a call for papers on issues of topical importance. Works received, and successful through peer review, will be compiled for publication as a special series.

In this first issue of the new year and volume thirty-eight, we present the works of authors who highlight the importance of adherence to reporting and safety in health settings, as well as the opportunity for insight gained through reflection on practice. Further, authors report on research undertaken to better understand a nurse-led service of early medication abortion. This issue's guest editorial by professors Juanita Sherwood, Roianne West, and colleagues from several Australian universities signals the beginning of a developing community of practice as First Nations nurses and midwives, educators, practitioners, and researchers. Here, in response to 2020's Black Lives Matter movement and recent dialogues regarding Australia Day, the authors urge us to consider the importance of our shared roles and responsibilities to continue to challenge racism and oppressive practices in Australian healthcare and society more broadly.

We thank all our authors for their hard work in bringing this issue together and the reviewers for their useful and informed critical appraisal.

To all, we thank you again for your ongoing support -not just to the journal, but for contributions which expand and build on the important evidence base underpinning the delivery of nursing, midwifery, health, and aged care not only in Australia, but internationally. As 2020 has shown us, nursing and midwifery is critical beyond borders, and in times when we might be limited in our travel, it is more important than ever to share our knowledge and experience.

\section{Casey Marnie}

Associate Editor, AJAN

University of South Australia, UniSA Clinical and Health Sciences, Rosemary Bryant AO Research Centre Adelaide, South Australia, Australia

\section{Dr Micah DJ Peters}

Editor-in-Chief, AJAN

University of South Australia, UniSA Clinical and Health Sciences, Rosemary Bryant AO Research Centre Adelaide, South Australia, Australia 